\title{
openheart Validation of the Intermountain Risk Score and Get with the Guidelines - Heart Failure Score in predicting mortality
}

\author{
Erik K Engelsgjerd, ${ }^{1}$ Catherine P Benziger, ${ }^{2}$ Benjamin D Horne (i) ${ }^{3,4}$
}

\begin{abstract}
- Additional supplemental material is published online only. To view, please visit the journal online (http://dx.doi.org/10. 1136/openhrt-2021-001722).

To cite: Engelsgjerd EK, Benziger CP, Horne BD. Validation of the Intermountain Risk Score and Get with the Guidelines-Heart Failure Score in predicting mortality. Open Heart 2021;8:e01722. doi:10.1136/ openhrt-2021-001722
\end{abstract}

Received 15 May 2021 Accepted 2 August 2021

A) Check for updates

(C) Author(s) (or their employer(s)) 2021. Re-use permitted under CC BY-NC. No commercial re-use. See rights and permissions. Published by BMJ.

${ }^{1}$ Department of Medicine, Des Moines University, Des Moines, Iowa, USA

${ }^{2}$ Heart and Vascular Center, Essentia Health, Duluth,

Minnesota, USA

${ }^{3}$ Intermountain Heart Institute, Intermountain Medical Center, Salt Lake City, Utah, USA ${ }^{4}$ Department of Medicine, Stanford University School of Medicine, Stanford, California, USA

Correspondence to Dr Benjamin D Horne; benjamin. horne@imail.org

\section{ABSTRACT}

Objective The Intermountain Risk Score (IMRS) was evaluated for validation as a mortality predictor and compared with the American Heart Association's Get With The Guidelines-Heart Failure (GWTG-HF) risk score in a rural heart failure (HF) population.

Background IMRS predicts mortality in general populations using common, inexpensive laboratory tests, patient age and sex, but requires validation in patients with HF.

Methods Individuals were selected from the GWTG-HF registry at Essentia Health. This included consecutive HF inpatients age $\geq 18$ years admitted July 2017-June 2019 . IMRS was calculated using sex-specific weightings of the complete blood count, basic metabolic profile, and age.

Results A total of 703 individuals (mean age: 74.12, $44.38 \%$ female) were studied. The 30 -day IMRS predicted 30-day mortality for both sexes (females $n=312: 0 R=1.19$ ( $95 \% \mathrm{Cl} 1.08$ to 1.32) per $+1, p<0.001$; males $n=391$ : $\mathrm{OR}=1.23(\mathrm{Cl} 1.12$ to 1.36) per $+1, \mathrm{p}<0.001)$. The GWTG$\mathrm{HF}$ risk score (only available in $\mathrm{n}=300,42.7 \%$ ) was independent of IMRS for 30-day mortality ( $\mathrm{OR}=1.11$ (Cl 1.06 to 1.16) per $+1, p<0.001$ ). Using thresholds in bivariate modelling, IMRS (high vs low risk, $O R=8.25(\mathrm{Cl}$ 2.19 to 31.09), $p=0.002$ ) and the GWTG-HF score (tertile 3 vs $1: 0 R=2.18(\mathrm{Cl} 0.84$ to 5.68$), p=0.11)$ independently predicted mortality. In multivariable analyses including covariables, IMRS (high vs low risk: $0 \mathrm{R}=6.69(\mathrm{Cl} 1.75$ to $25.60), p=0.005$ ) and the GWTG-HF score (tertile 3 vs 1 : $\mathrm{OR}=2.62$ ( $\mathrm{Cl} 0.96$ to 7.12 ), $\mathrm{p}=0.06$ ) remained predictors of mortality. Results were similar for 1-year mortality. Conclusions The IMRS and GWTG-HF scores predicted mortality of patients with HF in a large rural healthcare system. Future study of these scores as initial clinical risk estimators for evaluating their utility in improving patient health outcomes and increasing cost effectiveness is warranted.

\section{INTRODUCTION}

Heart failure (HF) affects 6 million adults in the USA and is associated with high morbidity and mortality. ${ }^{1}$ It has a high cost burden due to hospitalisations and persistent medical therapy with polypharmacy that are necessary to slow the disease's progression. While

\section{Key questions}

What is already known about this subject?

- Although many clinical decision tools exist that can be used to aid in the delivery of precision care, the utility of such scores remains limited because of the complexity of calculation by an already overburdened, time-scarce clinical workforce. The Intermountain Mortality Risk Score (IMRS) was derived from standardised, objective, commonly available laboratory factors in an urban healthcare population and predicts outcomes in many patients, including those with heart failure. IMRS was shown to be useful for guiding clinical decision-making in an urban population, but its predictive ability has not been tested in a rural setting.

What does this study add?

- In a rural heart failure population, both a clinical (ie, the Get With The Guidelines-Heart Failure risk score) and a laboratory-based (ie, IMRS) risk score predicted mortality after discharge from inpatient hospitalisation. While IMRS does not include all potential risk predictors, it provided at least a similar if not better ability to predict mortality as the heart failure-specific clinical score.

How might this impact on clinical practice?

- IMRS can be calculated in the background by the electronic health record and delivered electronically to the point of care without adding burden to clinical caregivers. This study suggests that IMRS, when used as a guide for clinical decision-making, may provide a reasonable alternative to other scores to identify patients with heart failure who require additional clinical attention to improve their health outcomes.

current medical therapies can improve the quality of life and reduce HF readmissions, the underlying cause of disease is rarely reversed or its progression halted by treatment. ${ }^{2} \mathrm{HF}$ prevalence has increased recently ${ }^{2}$ and, as more patients with HF live longer, the burden of HF treatment is growing. ${ }^{3}$ An urgent need exists to improve decision-making to more 
effectively and efficiently deliver patient-centred, personalised care that remains safe, timely and equitable.

Although predictive models for $\mathrm{HF}$ risk estimation exist, little is known about the impact of their use in patient care. Laboratory tests, such as a complete blood count $(\mathrm{CBC})$, are predictive of all cause mortality, ${ }^{4}$ and risk stratification scores using selected labs are helpful in a variety of conditions, including coronary artery disease (CAD) and transient ischaemic attacks. ${ }^{5-11}$ The Intermountain Risk Score (IMRS) is a clinical decisionsupport tool that uses common objective laboratory predictors like the CBC to estimate mortality risk. ${ }^{12-15}$ IMRS also predicts non-fatal outcomes including incident $\mathrm{HF}^{15}$ The score has multiple derivations using different weightings of its laboratory components depending on the outcome being predicted. ${ }^{12-16}$ IMRS guides clinician attention by supporting decision making and its use improved outcomes of patients hospitalised with HF. ${ }^{16}$ By using inexpensive, common laboratory tests, IMRS may provide a cost-effective quantification of HF risk that can be made readily available to clinicians through the electronic health record.

The American Heart Association's (AHA) Get With The Guidelines-HF (GWTG-HF) risk score is another tool developed to evaluate risk for patients with HF. In 2010, Peterson et $a l^{17}$ showed the GWTG-HF risk score predicted in-hospital mortality using seven variables: age, systolic blood pressure, blood urea nitrogen, heart rate, sodium, chronic obstructive pulmonary disease, and race. The GWTG-HF score predicted in-hospital mortality in patients with acute HF with preserved and with reduced left ventricular ejection fraction (LVEF). IMRS and GWTG-HF compared favourably in an urban California population, but their prediction ability in a rural population is unknown. ${ }^{14}$ This study evaluated IMRS for validation in a rural population of patients admitted with $\mathrm{HF}$ and compared its predictive ability to the HF-specific GWTG-HF risk score.

\section{METHODS}

\section{Study Population}

Data from the AHA GWTG-HF registry at Essentia Health were used for analysis. Essentia Health is a large rural healthcare system located in northern Minnesota, eastern North Dakota, and northern Wisconsin. GWTG-HF registry is the AHA's collaborative quality improvement programme that is demonstrated to improve adherence to evidence-based care of patients hospitalised with $\mathrm{HF}^{17}$ All consecutive adult inpatient admissions with primary diagnosis of $\mathrm{HF}$, including $\mathrm{HF}$ with reduced and preserved LVEF, at Essentia Health-St. Mary's between 1 July 2017 to 1 July 2019 were included.

\section{Study variables}

Baseline demographic variables included age, sex, race and comorbidity history including current smoker, hypertension, dyslipidaemia, family history of premature
$\mathrm{CAD}$, prior myocardial infarction (MI), prior HF, LVEF, diabetes mellitus and current haemodialysis. Laboratory data within 30 days prior to admission were used to calculate risk scores, including the $\mathrm{CBC}$ and comprehensive metabolic panel. Laboratory data included troponin, B-type natriuretic peptide, the CBC (haematocrit, red blood cell count, haemoglobin, white blood cell count, platelet count, mean corpuscular haemoglobin, mean corpuscular haemoglobin concentration, mean corpuscular volume, red cell distribution width and mean platelet volume), and the basic metabolic profile (BMP) (sodium, potassium, chloride, calcium, bicarbonate, blood urea nitrogen, creatinine, and glucose).

Variables contained in the GWTG-HF registry included medications prescribed at discharge, ECG, social support for the patient, education to the patient, blood pressure at discharge, and smoking cessation, among others. The GWTG-HF score was calculated using 7 variables, as noted above, ${ }^{17}$ and a patient's score was obtained by summing points assigned to each predictor, with final values ranging between 0 and 100. IMRS was calculated using sex-specific weightings for age and components from the $\mathrm{CBC}$ and BMP based on models previously derived in $>70000$ patients from Intermountain Healthcare. ${ }^{12} \mathrm{We}$ divided the subjects into three groups (low, moderate, high) based on previously validated, sex-specific cut offs for the risk scores. ${ }^{12}$

Study outcomes were 30-day mortality and 1-year mortality, which outcomes were previously defined, respectively, as those patients who died within 30 days of admission (including in-hospital deaths) and patients who died within 1 year of admission. The time until death and the time that non-decedents were lost to follow-up after hospitalisation were not available, thus logistic regression was used for analyses.

\section{Statistical considerations}

Inpatient data from hospitalizations between July 2017 and June 2019 in the Essentia Health system were compared between females and males using the $\chi^{2}$ test or Student's t-test for categorical or continuous variables, respectively, to describe baseline characteristics. Along with IMRS and the GWTG-HF score, the other study covariables were evaluated in univariate analyses to define the list of potential mortality predictors. Associations with mortality were assessed for categorical variables using the $\chi^{2}$ test (or the Fisher's exact test when appropriate). For continuous variables, the two-sample t-test (or Wilcoxon rank sum test when appropriate). IMRS and the GWTG-HF score were both evaluated as continuous variables and as categorical variables (IMRS using previously defined categories and GWTG-HF using tertiles).

Logistic regression models evaluated each risk score in univariable analysis for each endpoint and then bivariable models entering both scores simultaneously were constructed. Although intended to be used independent of other factors, the context of IMRS and GWTG-HF was also explored to determine their independent predictive 
Table 1 Baseline characteristics, by sex, of individuals selected for study from the GWTG-HF registry at Essentia Health (consecutive inpatients age $\geq 18$ years with HF admitted from July 2017 through June 2019)

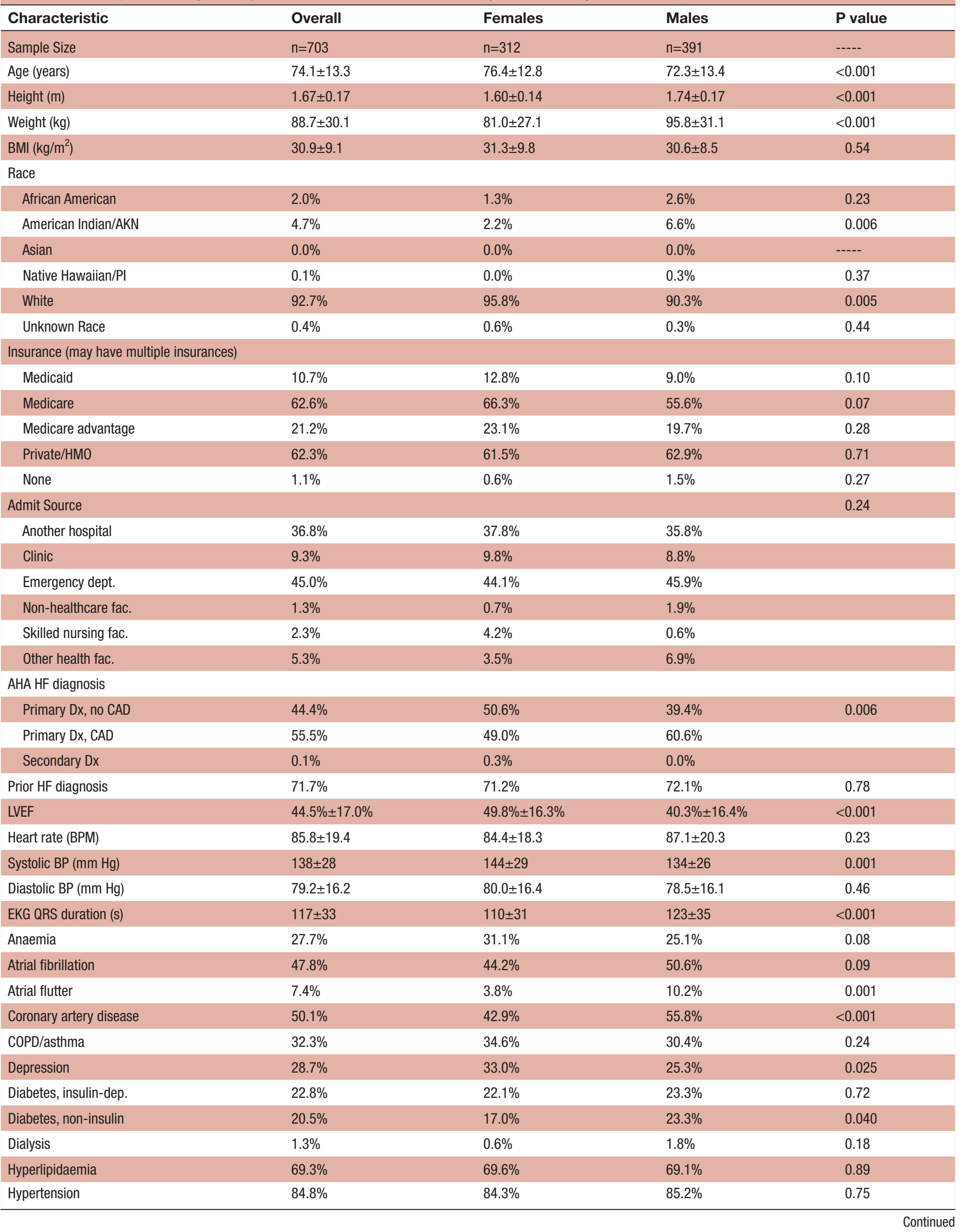




\begin{tabular}{lllll}
\hline Table 1 Continued & & & & \\
\hline Characteristic & Overall & Females & Males & P value \\
\hline Pacemaker & $11.2 \%$ & $13.1 \%$ & $9.7 \%$ & 0.15 \\
\hline Peripheral vascular dis. & $15.2 \%$ & $14.7 \%$ & $15.6 \%$ & 0.75 \\
\hline Prior CABG & $19.9 \%$ & $14.7 \%$ & $24.0 \%$ & 0.002 \\
\hline Prior MI & $24.5 \%$ & $19.6 \%$ & $28.4 \%$ & 0.007 \\
\hline Prior PCl & $30.2 \%$ & $27.2 \%$ & $32.5 \%$ & 0.13 \\
\hline Rales & $21.8 \%$ & $23.4 \%$ & $20.5 \%$ & 0.35 \\
\hline Renal insufficiency & $26.3 \%$ & $22.8 \%$ & $29.2 \%$ & 0.06 \\
\hline Sleep-disord. breathing & $13.2 \%$ & $9.9 \%$ & $15.9 \%$ & 0.021 \\
\hline Smoking history & $18.2 \%$ & $14.4 \%$ & $21.2 \%$ & 0.020 \\
\hline Stroke & $18.6 \%$ & $19.2 \%$ & $18.2 \%$ & 0.72 \\
Valvular heart disease & $25.3 \%$ & $26.6 \%$ & $24.3 \%$ & 0.49 \\
\hline BNP $(\mathrm{pg} / \mathrm{mL})$ & $1468 \pm 1439$ & $1438 \pm 1541$ & $1492 \pm 1352$ & 0.64 \\
\hline Troponin (ng/mL) & $0.44 \pm 2.57$ & $0.26 \pm 1.40$ & $0.58 \pm 3.22$ & 0.12 \\
\hline Troponin, peak (ng/mL) & $1.31 \pm 11.0$ & $0.59 \pm 3.27$ & $1.89 \pm 14.54$ & 0.14 \\
\hline
\end{tabular}

AKN, Alaskan native; BMI, body mass index; BNP, B type natriuretic peptide; BP, blood pressure; BPM, beats per minute; CABG, coronary artery bypass graft; CAD, coronary artery disease; COPD, chronic obstructive pulmonary disease; GWTG-HF, Get With The Guidelines-Heart Failure; HF, heart failure; IMRS, Intermountain Mortality Risk Score; LVEF, left ventricular ejection fraction; MI, myocardial infarction; PCI, percutaneous coronary intervention (including balloon angioplasty and coronary stenting); PI, pacific islander.

ability in the setting of other predictors such as risk factors, medications and demographics (not all patients had LVEF data available at baseline, thus separate modelling was performed to evaluate the effect of LVEF on the risk scores). Therefore, other predictors of mortality with at least a moderate level of association $(\mathrm{p}<0.10)$ were entered into multivariate logistic regression models and removed stepwise using forward and backward

\begin{tabular}{|c|c|c|c|c|}
\hline Characteristic & Overall & Females & Males & $P$ value \\
\hline GWTG-HF score $(n=300)$ & $42.0 \pm 8.7$ & $40.6 \pm 8.8$ & $43.3 \pm 8.5$ & 0.008 \\
\hline 30-day IMRS* (n=703) & * & $16.7 \pm 3.9$ & $16.0 \pm 3.7$ & 0.012 \\
\hline 1-year IMRS* $(n=703)$ & * & $14.5 \pm 3.5$ & $14.7 \pm 3.5$ & 0.43 \\
\hline $\mathrm{RBC}\left(\times 10^{6} / \mu \mathrm{L}\right)$ & $4.03 \pm 0.82$ & $3.88 \pm 0.73$ & $4.15 \pm 0.87$ & $<0.001$ \\
\hline Haemoglobin (g/dL) & $11.7 \pm 2.3$ & $11.3 \pm 2.0$ & $12.1 \pm 2.4$ & $<0.001$ \\
\hline Haematocrit (\%) & $36.6 \pm 6.7$ & $35.5 \pm 5.9$ & $37.5 \pm 7.1$ & $<0.001$ \\
\hline WBC $\left(\times 10^{9} / L\right)$ & $8.96 \pm 3.59$ & $8.84 \pm 3.28$ & $9.05 \pm 3.82$ & 0.44 \\
\hline MCV (fL) & $91.2 \pm 7.7$ & $91.3 \pm 7.8$ & $91.1 \pm 7.6$ & 0.84 \\
\hline RDW (\%) & $15.8 \pm 2.4$ & $15.8 \pm 2.4$ & $15.9 \pm 2.5$ & 0.57 \\
\hline $\mathrm{MCH}(\mathrm{pg})$ & $30.8 \pm 2.7$ & $30.7 \pm 2.6$ & $30.9 \pm 2.7$ & 0.22 \\
\hline MCHC (g/dL) & $32.1 \pm 1.5$ & $31.9 \pm 1.5$ & $32.2 \pm 1.5$ & 0.002 \\
\hline MPV (fL) & $9.77 \pm 1.12$ & $10.34 \pm 1.14$ & $9.14 \pm 0.71$ & 0.015 \\
\hline Sodium (mmol/L) & $136.9 \pm 4.8$ & $137.0 \pm 4.7$ & $136.9 \pm 4.9$ & 0.64 \\
\hline Potassium (mmol/L) & $4.20 \pm 0.61$ & $4.15 \pm 0.61$ & $4.24 \pm 0.61$ & 0.06 \\
\hline Calcium (mg/dL) & $9.04 \pm 0.80$ & $9.16 \pm 0.69$ & $8.94 \pm 0.86$ & $<0.001$ \\
\hline Bicarbonate (mmol/L) & $25.0 \pm 4.8$ & $25.2 \pm 5.0$ & $24.8 \pm 4.6$ & 0.28 \\
\hline Creatinine (mg/dL) & $1.49 \pm 0.89$ & $1.30 \pm 0.65$ & $1.63 \pm 1.02$ & $<0.001$ \\
\hline BUN (mg/dL) & $31.4 \pm 20.1$ & $29.9 \pm 20.2$ & $32.6 \pm 19.9$ & 0.07 \\
\hline Glucose (mg/dL) & $143 \pm 69$ & $136 \pm 63$ & $149 \pm 72$ & 0.015 \\
\hline
\end{tabular}

*This risk score was developed with sex-specific weights for risk components; thus, an overall mean is not reported.

GWTG-HF, Get With The Guidelines-Heart Failure; IMRS, Intermountain Mortality Risk Score. 
Table 3 Mortality outcomes in tertiles of the Get With The Guidelines-Heart Failure (GWTG-HF) score ( $\mathrm{n}=300)$ and in previously established categories of the 30-day and 1-year versions of Intermountain Mortality Risk Score (IMRS) $(n=703)^{12}$

\begin{tabular}{|c|c|c|c|}
\hline Outcome/risk score & Overall & Females & Males \\
\hline \multicolumn{4}{|l|}{ GWTG-HF $(n=300)$} \\
\hline Tertile 2 & $6.1 \%(n=99)$ & $6.1 \%(n=49)$ & $6.0 \%(n=50)$ \\
\hline Tertile 3 & $23.1 \%(n=104)$ & $22.5 \%(n=40)$ & $23.4 \%(n=64)$ \\
\hline \multicolumn{4}{|l|}{ 30-day IMRS } \\
\hline Low risk & $4.6 \%(n=217)$ & $2.5 \%(n=79)$ & $5.8 \%(n=138)$ \\
\hline Moderate risk & $11.0 \%(n=310)$ & $13.3 \%(n=158)$ & $8.6 \%(n=152)$ \\
\hline High risk & $21.0 \%(n=176)$ & $20.0 \%(\mathrm{n}=75)$ & $21.8 \%(n=101)$ \\
\hline Tertile 1 & $16.5 \%(\mathrm{n}=97)$ & $20.8 \%(n=53)$ & $11.4 \%(n=44)$ \\
\hline Tertile 2 & $23.2 \%(n=99)$ & $22.4 \%(n=49)$ & $24.0 \%(n=50)$ \\
\hline Tertile 3 & $37.5 \%(n=104)$ & $35.0 \%(n=40)$ & $39.1 \%(n=64)$ \\
\hline p-trend* & $<0.001$ & 0.13 & 0.001 \\
\hline \multicolumn{4}{|l|}{ 1-year IMRS } \\
\hline Low risk & $7.4 \%(n=68)$ & $5.6 \%(n=18)$ & $8.0 \%(n=50)$ \\
\hline Moderate risk & $25.2 \%(n=353)$ & $25.8 \%(n=132)$ & $24.9 \%(n=221)$ \\
\hline High risk & $42.2 \%(n=282)$ & $40.1 \%(n=162)$ & $45.0 \%(n=120)$ \\
\hline p-trend* & $<0.001$ & $<0.001$ & $<0.001$ \\
\hline
\end{tabular}

For GWTG-HF, tertiles constituted patients with scores of $\leq 37$ (tertile 1), 38-45 (tertile 2), and $\geq 46$ (tertile 3). For IMRS, 30-day risk categories for females and males were, respectively, low-risk: $\leq 14$ and $\leq 14$; moderate risk: 15-19 and 15-18; high risk: $\geq 20$ and $\geq 19$; while 1-year risk categories for females and males were, respectively, low risk: $\leq 8$ and $\leq 10$; moderate risk: 9-14 and 11-16; high risk: $\geq 15$ and $\geq 17$. ${ }^{*}$-trend across tertiles 1, 2, and 3 of GWTG-HF or across low, moderate, and high-risk categories of IMRS. IMRS, Intermountain Mortality Risk Score.

elimination procedures. Only variables that had a significant association $(p<0.05)$ were retained for the final model. The area under the receiver operator curve $(\mathrm{C}$ statistic) was used to test the model's performance. A C statistic of 0.5 indicates a model predicts the outcome no better than random change and a $\mathrm{C}$ statistic of 1 indicated a model has perfect discrimination. Sensitivity, specificity and positive and negative predictive values were calculated to evaluate the performance of the risk scores. SPSS V.26.0 (IBM, SPSS) was used for data analysis with $\mathrm{p} \leq 0.05$ defined as statistically significant.

\section{RESULTS}

There were 703 patients included in the cohort, 312 female, 391 male, mean age: $74.1 \pm 13.3$ years. Association of IMRS with additional demographics, cardiac comorbidities and additional risk factors are shown in table 1. Overall, $71.7 \%$ of patients had a previous diagnosis of HF, $84.8 \%$ had baseline hypertension, $22.8 \%$ insulin dependent diabetes, $18.2 \%$ smoking, $50.1 \%$ CAD, $24.5 \%$ prior MI and $1.3 \%$ haemodialysis.
Women were generally older $(76.4 \pm 12.8$ vs $72.3 \pm 13.4$, $\mathrm{p}<0.001)$, shorter $(1.60 \pm 0.14$ vs $1.74 \pm 0.17, \mathrm{p}<0.001)$ and weighed less than men $(81.0 \pm 27.1$ vs $95.8 \pm 31.1, \mathrm{p}<0.001)$. Women were less likely to have a prior HF diagnosis (71.2\% vs 72.1, $\mathrm{p}=0.78)$, presented with higher systolic blood pressure $(144 \pm 29$ vs $134 \pm 26, \mathrm{p}=0.001)$, and were less likely to have CAD (42.9\% vs $55.8 \%, \mathrm{p}<0.001)$. Furthermore, women were less likely to have prior coronary artery bypass grafting $(14.7 \%$ vs $24.0 \%, \mathrm{p}=0.002)$ and prior MI ( $19.6 \%$ vs $28.4 \%, \mathrm{p}=0.007)$. Women were more likely to have depression than men $(33.0 \%$ vs $25.3 \%, \mathrm{p}=0.025)$. Baseline creatinine was $1.49 \pm 0.89$ (women: $1.30 \pm 0.65$, men: $1.63 \pm 1.02 ; \mathrm{p}<0.001$ ), which was significantly above normal. Also, the mean haemoglobin was lower in men than for women in relation to normal values (women: 11.3 \pm 2.0 , men: $12.1 \pm 2.4 ; \mathrm{p}<0.001$ ). All other laboratory data are shown in table 2 .

Overall 30-day mortality was $11.5 \%$ and, based on IMRS, $4.6 \%$ of the low-risk group, $11.0 \%$ of the moderate-risk group, and $21 \%$ of the high-risk group died $(\mathrm{p}<0.001)$. This trend continued in the 1-year mortality outcomes 
A)

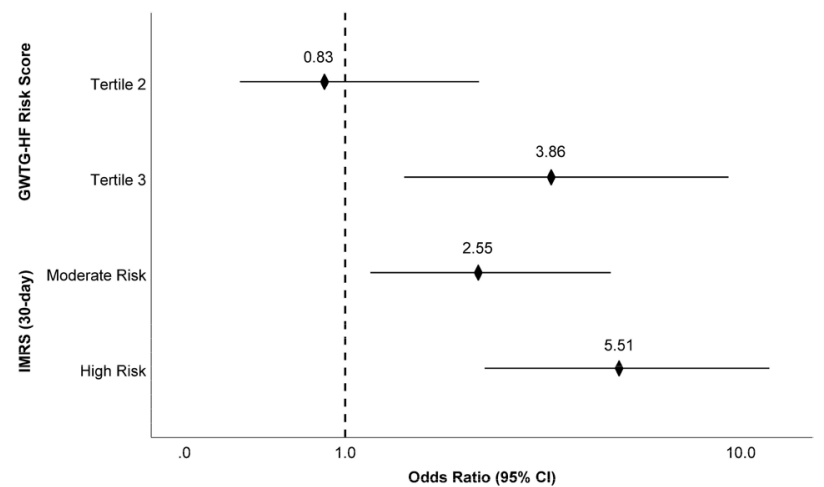

B)

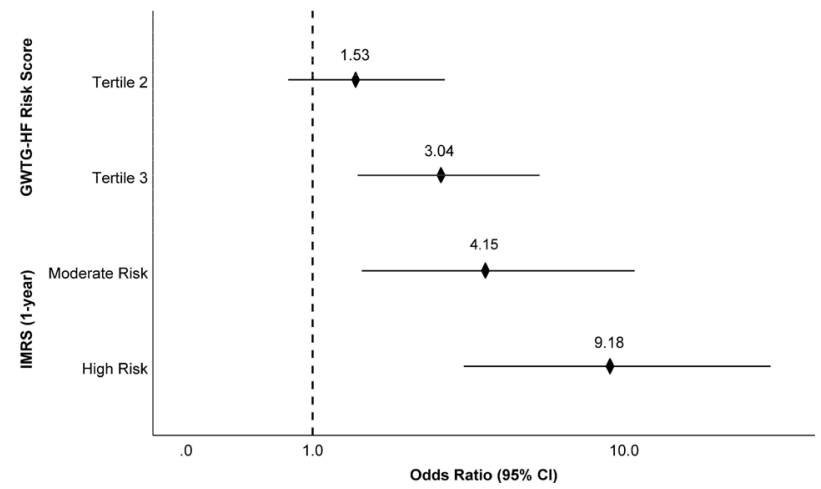

Figure 1 Mortality odds by risk score. Forest plots of the ORs with $95 \%$ Cls for the univariable associations with: (A) 30-day mortality and (B) 1-year mortality, for the Get With The Guidelines-Heart Failure (GWTG-HF) risk score (tertiles 2 and 3 compared with tertile $1, n=300$ ) and Intermountain Mortality Risk Score (IMRS) (moderate and high risk compared with low risk, $n=703$ ). The $x$-axis is displayed on a logarithmic scale.

where the 1-year overall mortality was $30 \%$ and IMRS identified that $7.4 \%$ died in the low-risk group, $25.2 \%$ in the moderate-risk group, and $42.2 \%$ in the high-risk category $(p<0.001)$. Table 3 shows the percentage of mortality for the GWTG-HF risk score, which also was associated with mortality at both time points. The table further provides mortality results for females and males separately.

The univariate associations of each score are provided in figure 1 for 30-day and 1-year mortality, with a broader set of data and specific values shown in online supplemental table S1. Using continuous variables, the GWTG-HF score was validated for both 30-day mortality (OR: 1.11 per +1 score (95\% CI 1.06 to 1.16$), \mathrm{p}<0.001$ ) and 1-year mortality (OR 1.08 (CI 1.04 to 1.11 ), p<0.001). IMRS strongly predicted risk in both time frames for both men (30-day mortality: OR 1.23 (CI 1.12 to 1.36 ), $\mathrm{p}<0.001$; 1-year mortality: OR 1.28 (CI 1.18 to 1.38 ), $\mathrm{p}<0.001$ ) and women (30-day mortality: OR 1.19 (CI $1.08,1.32$ ), $\mathrm{p}<0.001$; 1-year mortality: OR 1.14 (CI 1.06 to $1.23), \mathrm{p}<0.001)$.

Joint evaluation of IMRS and the GWTG-HF risk score showed generally that the two scores were complementary, with each score being independently predictive of mortality in bivariable analysis (table 4). Unfortunately, the GWTG-HF had substantial missing data; thus, the sample size was $\mathrm{n}=300$ for all analyses including the
GWTG-HF risk score. Evaluation of IMRS within each tertile of the GWTG-HF risk score extended this joint evaluation, showing added value of calculating both scores for most patients and especially for those in the first and second tertiles (online supplemental table S2 and figure S1).

Subanalyses of the subset with LVEF data available at baseline $(n=684)$ showed that the associations of both IMRS and the GWTG-HF score with 30-day and 1-year mortality improved $1 \%-5 \%$ when LVEF was added to the model. The only exception to this was in analyses entering categorical IMRS and GWTG-HF variables along with LVEF wherein the associations of IMRS improved $4 \%-5 \%$ but those of the GWTG-HF score declined $21 \%$ (30-day mortality) and 10\% (1-year mortality).

\section{DISCUSSION}

In a rural healthcare setting, IMRS and the GWTG-HF risk scores were separately associated with 30-day and 1-year mortality in patients initially hospitalised for HF. Intended to be used as self-contained risk metrics, these risk scores were also shown to be independent, complementary predictors of mortality when examined together. The scores predicted mortality both as continuous variables and when categorised, with IMRS stratifying risk especially well within the first and second tertiles of the GWTG-HF risk score.

IMRS was originally developed as a clinical application using the association of the CBC and BMP testing components, in addition to sex and age, with mortality. ${ }^{12}$ This was done without consideration of other risk factors, morbidity, ongoing disease states, or any additional information about the patient's health, ${ }^{12}$ including frequently discussed HF-specific variables, such as LVEF, patient follow-up information, patient social support, B-type natriuretic peptide, and others. As IMRS was further studied it was found that, despite conservative correction for multiple comparisons, it predicted multiple different disease states and risk factors that may lead to mortality ${ }^{14-16}$ IMRS was associated with history of HF, mean LVEF, admission for HF after initial diagnosis, incident HF, MI diagnosis, incident MI, atrial fibrillation and atrial flutter, and chronic obstructive pulmonary disease; with each of these associations being replicated in a follow-up, larger, independent patient population. ${ }^{15}$ That study showed the ability of IMRS to stratify risk levels for common clinical cardiovascular conditions, and subsequent study of patients with HF in an urban population showed IMRS was independent of the GWTG-HF score. ${ }^{14}$ The present study expands this to a rural HF population.

When evaluating IMRS and GWTG-HF, it is important to consider the risk scores' clinical use. A truly effective tool has the ability to consider a large number of clinical variables related to patient management simultaneously. At the same time, the score should illuminate seemingly benign clinical data that may contain previously unidentified risk assessment variables. Further, clinicians should 
Table 4 ORs and 95\% Cls in bivariable modelling entering both the Get With The Guidelines-Heart Failure (GWTG-HF) and Intermountain Mortality Risk Scores (IMRS) into the same model, and in multivariable modelling entering the two scores and adjusting for other potential risk predictors*

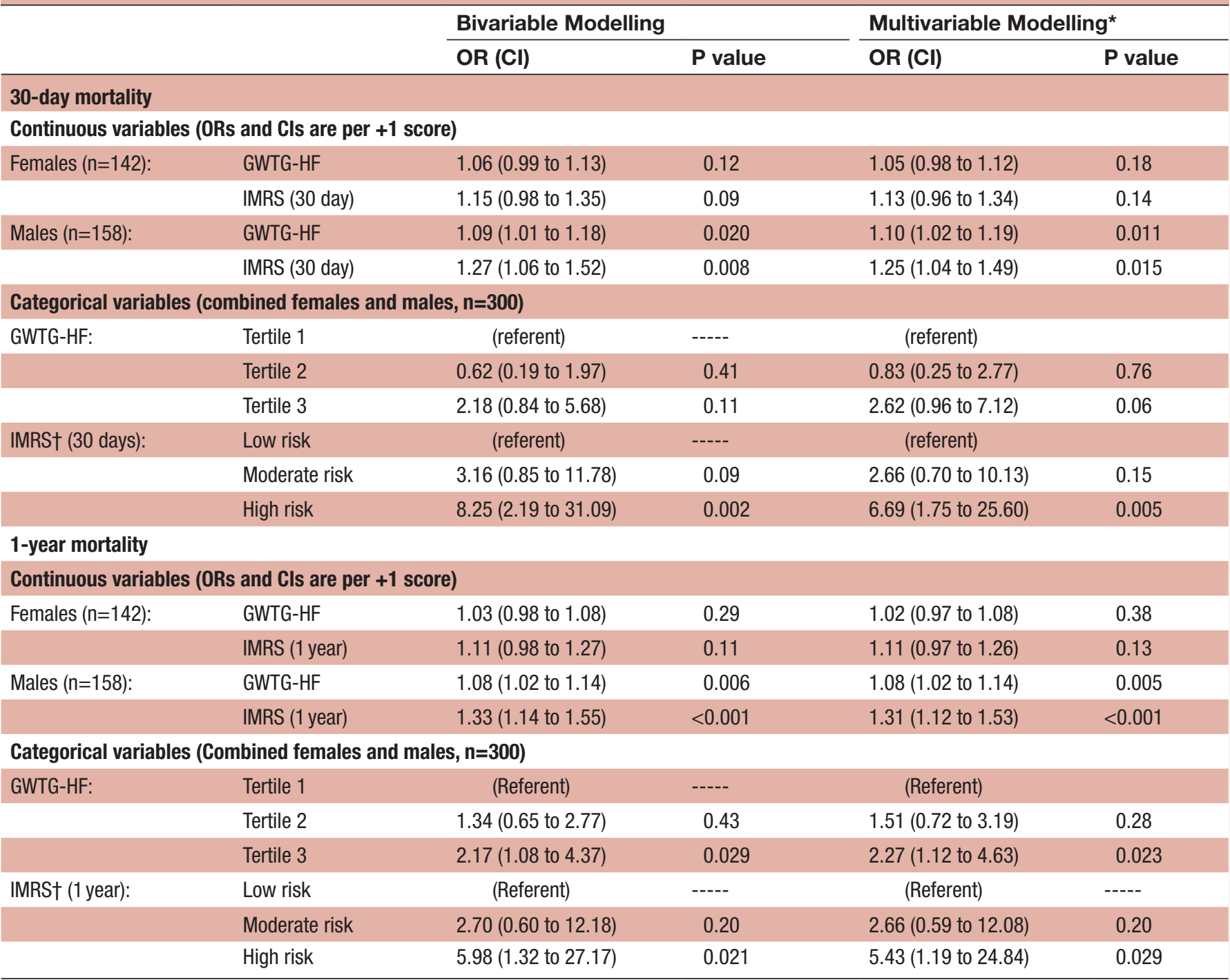

All of these analyses only evaluated $n=300$ patients (or sex-specific subsets) for whom GWTG-HF was available.

*Study covariables considered for entry into multivariable models were: sex, race (African American, American Indian/Alaska Native, Asian, Native Hawaiian/Pacific Islander, White, unknown), admit source (another hospital, clinic, emergency department, non-healthcare facility, skilled nursing facility, other health facility), insurance type (private/HMO, Medicaid, Medicare, Medicare advantage, none), prior heart failure (HF) diagnosis, anaemia, atrial fibrillation, atrial flutter, chronic obstructive pulmonary disease/asthma, coronary artery disease (CAD), depression, hyperlipidaemia, hypertension, insulin-dependent diabetes, non-insulin-dependent diabetes, dialysis, pacemaker, peripheral vascular disease (PVD), prior coronary artery bypass grafting, prior myocardial infarction, prior percutaneous coronary intervention, rales, renal insufficiency, sleep disordered breathing, smoking history, stroke, valvular heart disease, EKG QRS duration, ACE inhibitor, aldosterone antagonist, beta-blocker, bumex, demadex, edecrin, factor Xa inhibitor, hydralazine nitrate, lasix, loop diuretics, metolazone, and type of heart failure diagnosis (primary diagnosis without $C A D$, primary diagnosis with $C A D$, or secondary diagnosis). Multivariable models entered the following covariables in the final models (in addition to the two risk score variables): for 30-day Mortality: PVD and beta-blocker; for 1year mortality: insulin-dependent diabetes, hyperlipidaemia, history of smoking, and beta-blocker.

tIMRS values were categorised into low risk, moderate risk, and high risk based on criteria from 2009 for 30 -day and 1 -year risk scores. ${ }^{12}$ Once the categories were assigned based on sex-specific and time frame-specific criteria, the data for females and males could be combined because the criteria for assigning thresholds of risk were the same for both sexes although the numeric distribution of the scores and the actual thresholds were different.

be able to receive the score and have clear actions to take in response to different risk levels, without having to invest more than a few seconds of their time to view the electronic health record. Both risk scores have the value of accessibility, both being able to be immediately used in the medical setting. One draw back of the GWTG-HF is its focus on a specific population: patients with diagnoses of HF, while one study did look at the predictive ability of the GWTG-HF score to predict undifferentiated patients in the cardiac intensive care unit. ${ }^{4}$ The GWTG-HF score was also obtained retrospectively in the registry and was not able to be calculated in the entire cohort due to 
missing variables, such as systolic blood pressure, heart rate, chronic obstructive pulmonary disease, or race.

Finally, comorbidities are used in the GWTG-HF risk score assessment, making the score less flexible in the situation that patients improve from the standpoint of their HF. If patients improve with treatment, the GWTG-HF risk score stays fixed while IMRS is more flexible in that it can change dynamically because it is based on basic laboratory values. If patients are adherent to their treatment, then their risk score can decrease. Inversely, if a patient has poor adherence to their medication or if the medications are ineffective, this can be tracked more readily.

IMRS uses very common laboratory tests (CBC, BMP) and was developed among a very large general patient population. In previous studies, IMRS predicted mortality in two general patient samples, ${ }^{12}$ the National Health and Nutrition Examination Survey III population, ${ }^{12}$ people free of cardiovascular diseases, ${ }^{13}$ two samples of coronary angiography patients, ${ }^{12} 15$ patients with HF at Stanford University, ${ }^{14}$ and patients with $\mathrm{HF}$ at Intermountain Healthcare. ${ }^{16}$ IMRS may be of interest for clinical use for general practictioners who provide care for common cardiovascular conditions and other illnesses that lead to mortality, and also for cardiologists who care for patients with $\mathrm{HF}, \mathrm{CAD}$, and other higher acuity conditions. Broad applicability at low financial and clinical costs are the overarching benefits of IMRS.

\section{Limitations}

This study was conducted with 703 patients at a single medical centre using an observational design. Potential issues with confounding and unobserved variables may be in play. Evaluation of IMRS in a larger sample size of people from rural locales would aid in strengthening the validation and clarifying some of the borderline results. This is especially the case in analyses of IMRS with the GWTG-HF risk score because the second score was only available in a subfraction of patients. Further exploration of the apparent independent, complementary nature of the two scores is needed. A strength of the study, though, is that IMRS and the GWTG-HF score were developed to be used without explicit inclusion of other variables; thus, basic analyses of these individually provide a real-world evaluation of how the scores would be used in practice. It may be that other data elements such as NYHA classification of HF stage would have aided in assessing the full extent of risk in this population, but NYHA class was not available in the study dataset or its electronic clinical source data. Further evaluations using NYHA class and potentially other HF-associated predictors could improve risk prediction in this and similar populations.

\section{CONCLUSION}

IMRS predicted 30-day and 1-year mortality for patients with HF in a large rural healthcare system. The GWTG-HF risk score also predicted 30-day and 1-year mortality in this population, but analysis was limited due to a lack of variables in this retrospective study. When looking at the modern clinical world, it is important to find tools that evaluate patient information and provide usable, reliable data and interface seamlessly with the electronic health record for all patients. IMRS uses simple variables that most patients have on admission, therefore making it very easy and useful to practitioners. With both the IMRS and GWTG-HF being independently predictive of mortalilty, it is warranted to continue study of approaches that use the scores for initial clinical risk estimation to personalise and optimise healthcare, improving patient health outcomes and increasing cost effectiveness of care.

\section{Twitter Benjamin D Horne @DrBenjaminHorne}

Contributors CPB and BDH had full access to all study data and take responsibility for the integrity of the data and the accuracy of the data analysis. CPB and BDH had authority over manuscript preparation and the decision to submit the manuscript for publication. Conception and design: $\mathrm{CPB}, \mathrm{BDH}$; acquisition, analysis, or interpretation of data: all authors; drafting of the manuscript: EKE; critical revision for important intellectual content: $\mathrm{CPB}, \mathrm{BDH}$; final approval of the submitted manuscript: all authors; agreement to be accountable for all aspects of the work: $\mathrm{CPB}, \mathrm{BDH}$.

Funding The authors thank the Essentia Health Foundation grant for funding support. The authors take full responsibility for manuscript content, with the funding sources having no role in the design, conduct, or analysis of the study, or in writing or revising the manuscript.

Competing interests $\mathrm{BDH}$ is an inventor of clinical decision tools that are licensed to CareCentra and Alluceo, the PI of grants involving clinical decision tools that were funded by the Intermountain Research and Medical Foundation, CareCentra, GlaxoSmithKline, and AstraZeneca, a member of the scientific advisory board of Labme.ai, and a co-investigator on a grant funded by the Patient-Centered Outcomes Research Institute (PCORI). The authors declare that no other conflicts of interest or disclosures exist.

Patient consent for publication Not required.

Ethics approval This study was approved by the Essentia Health Institutional Review Board as a retrospective data review study with no patient contact that was exempted from obtaining informed consent. The ID number of the IRB protocol for the study is EH19603. No patient or public involvement was included in the design, conduct, or reporting/dissemination of this study.

Provenance and peer review Not commissioned; externally peer reviewed.

Data availability statement Data are available upon reasonable request. The data underlying this article cannot be shared publicly due to US privacy laws. The data will be shared contractually on reasonable request to Dr. Benziger at Catherine. Benziger@EssentiaHealth.org.

Open access This is an open access article distributed in accordance with the Creative Commons Attribution Non Commercial (CC BY-NC 4.0) license, which permits others to distribute, remix, adapt, build upon this work non-commercially, and license their derivative works on different terms, provided the original work is properly cited, appropriate credit is given, any changes made indicated, and the use is non-commercial. See: http://creativecommons.org/licenses/by-nc/4.0/.

ORCID iD

Benjamin D Horne http://orcid.org/0000-0002-2656-0263

\section{REFERENCES}

1 Roger VL, Go AS, Lloyd-Jones DM, et al. Executive summary: heart disease and stroke statistics--2012 update: a report from the American Heart Association. Circulation 2012;125:188-97.

2 Cubbon RM, Gale CP, Kearney LC, et al. Changing characteristics and mode of death associated with chronic heart failure caused by left ventricular systolic dysfunction: a study across therapeutic eras. Circ Heart Fail 2011;4:396-403.

3 Stewart S, Ekman I, Ekman T, et al. Population impact of heart failure and the most common forms of cancer: a study of 1162 309 hospital cases in Sweden (1988 to 2004). Circ Cardiovasc Qual Outcomes 2010;3:573-80. 
4 Lyle M, Wan S-H, Murphree D, et al. Predictive value of the get with the guidelines heart failure risk score in unselected cardiac intensive care unit patients. J Am Heart Assoc 2020;9:e012439.

5 Anderson JL, Ronnow BS, Horne BD, et al. Usefulness of a complete blood count-derived risk score to predict incident mortality in patients with suspected cardiovascular disease. Am J Cardiol 2007:99:169-74.

6 Wilson PW, D'Agostino RB, Levy D, et al. Prediction of coronary heart disease using risk factor categories. Circulation 1998;97:1837-47.

7 Neaton JD, Wentworth D. Serum cholesterol, blood pressure, cigarette smoking, and death from coronary heart disease. overall findings and differences by age for 316,099 white men. multiple risk factor intervention trial Research Group. Arch Intern Med 1992:152:56-64.

8 Dankner R, Goldbourt U, Boyko V, et al. Predictors of cardiac and noncardiac mortality among 14,697 patients with coronary heart disease. Am J Cardiol 2003;91:121-7.

9 D'Agostino RB, Russell MW, Huse DM, et al. Primary and subsequent coronary risk appraisal: new results from the Framingham study. Am Heart J 2000;139:272-81.

10 Rothwell PM, Giles MF, Flossmann E, et al. A simple score (ABCD) to identify individuals at high early risk of stroke after transient ischaemic attack. Lancet 2005;366:29-36.
11 Liao L, Kong DF, Shaw LK, et al. A new anatomic score for prognosis after cardiac catheterization in patients with previous bypass surgery. J Am Coll Cardiol 2005;46:1684-92.

12 Horne BD, May HT, Muhlestein JB, et al. Exceptional mortality prediction by risk scores from common laboratory tests. Am J Med 2009;122:550-8

13 Horne BD, Anderson JL, Muhlestein JB, et al. Complete blood count risk score and its components, including RDW, are associated with mortality in the JUPITER trial. Eur J Prev Cardiol 2015;22:519-26.

14 Boralkar KA, Kobayashi Y, Moneghetti KJ, et al. Improving risk stratification in heart failure with preserved ejection fraction by combining two validated risk scores. Open Heart 2019;6:e000961.

15 Horne BD, May HT, Kfoury AG, et al. The intermountain risk score (including the red cell distribution width) predicts heart failure and other morbidity endpoints. Eur J Heart Fail 2010;12:1203-13.

16 Horne BD, Roberts CA, Rasmusson KD, et al. Risk score-guided multidisciplinary team-based care for heart failure inpatients is associated with lower 30-day readmission and lower 30-day mortality. Am Heart J 2020;219:78-88.

17 Peterson PN, Rumsfeld JS, Liang L, et al. A validated risk score for in-hospital mortality in patients with heart failure from the American Heart Association get with the guidelines program. Circ Cardiovasc Qual Outcomes 2010;3:25-32. 